\title{
Exploring robust, intuitive and emergent physical human-robot interaction with the humanoid robot Acroban
}

\author{
Pierre-Yves Oudeyer \\ INRIA, France
}

\author{
Olivier Ly \\ INRIA/University of Bordeaux, France
}

\author{
Pierre Rouanet \\ INRIA, France
}

\begin{abstract}
We present how a humanoid robot, called Acroban, allows whole-body robust, natural and intuitive physical interaction with both adults and children. These physical human-robot interaction are made possible through the combination of several properties of Acroban: 1) it is whole-body compliant thanks to variable impedance control and also thanks to the use of elastics and springs; 2) it has a bio-inspired vertebral column allowing more flexibility in postural and equilibrium control; 3 ) it is lightweight; 4) it has simple low-level controllers that leverage the first three properties. Moreover, the capabilities for physical humanrobot interaction that we show are not using a model of the human, and in this sense are "model free": 1) the capability of the robot to keep its equilibrium while being manipulated or pushed by humans is a result of the intrinsic capability of the whole body to absorb unpredicted external perturbations; 2) the capability of leading Acroban by the hand is an emergent human-robot interface made possible by the self-organizing properties of the body and its low-level controllers and was observed a posteriori only after the robot was conceived and without any initial plan to make this possible. Finally, an originality of Acroban is that is is made with relatively low-cost components which lack of precision is counterbalanced with the robustness due to global geometry and compliance.
\end{abstract}

\section{INTRODUCTION}

Personal robots, used for physical, cognitive or social assistance, as well as for entertainment, are predicted to arrive massively in our homes in coming decades. Yet, before this vision becomes a reality, many important challenges need to be addressed. Among them, safe and intuitive physical interaction is essential. Who could imagine an assistive robot which should not be touched? Physical assistance obviously implies physical contact. As far as cognitive or social assistance is concerned, how could a personal robot be accepted if children around would be permanently forbidden to approach it, give it a tap in the back, or shoot a foot ball at it? Yet, at the exception of simple single task robots such as vaccum cleaners or entertainment toy robots such as the Sony AIBO, many existing robots do not permit physical interaction, often risking to harm the human and/or the robot due to the combination of their weight, fragility and power. This is particularly the case for humanoid robots, complex enough for achieving useful complex and diverse motor tasks, but so far mostly lacking the capabilities to be at the same time robust to unknown external perturbations (e.g. Honda's ASIMO [1], HRP-4 [2] or Aldebaran Robotics' Nao), lighweight, affordable, and both intuitive and playful to interact with.
In this article, we study how a novel humanoid platform, called Acroban (see [3], [4]), addresses those challenges and opens new possibilities for rich, robust, intuitive and even playful compliant human-humanoid physical interaction. To our knowledge, Acroban may be the first light-weight lowcost humanoid robot allowing compliant full-body physical interaction, with human children in particular (see figure 1), capable of robust dynamic balancing and walking in the face of potentially large unknown external perturbations. Heavily relying on the use of adequate materials and morphology, as well as on the concept of morphological computation [5], [6], Acroban is not only robust and safe to external perturbations, but its natural dynamics spontaneously generates emergent analogic interfaces such as one allowing to drive it by the hand, like we are used to do with a little child. Those various properties of Acroban are the result of combining several features:

- Bio-inspired vertebral column and morphology: In contrast with most humanoid robots which have a rigid "box" in place of the torso, Acroban has a multiarticulated vertebral column inspired by human morphology [7]. It has been shown that the human vertebral column is essential for keeping one own's balance, in particular during dynamic walking [7]. Likewise, we argue that a vertebral column opens stimulating possibilies in humanoid robotics;

- Full-body compliance: Both the actuators and the very structure of Acroban are highly compliant, i.e. they can be made to bend smoothly when external forces are applied to them and absord them. In particular, motors are controlled in variable impedance [8], [9];

- Light-weightedness and the possibility to acquire online new movements: Designing robot movements is an extremely difficult task, especially when it comes to dynamic motor primitives for balancing, walking and human-robot interaction, due to the complexity of physics and unpredictability of humans. One possible approach is to use very high-technology actuators and structures allowing such a precision and determinism that it becomes possible to make analytical mathematical models and simulations of the robot's physics and derive theoretically controllers [2], [10]. Yet, this entails using very expensive 
and typically rather large, heavy and fragile actuators which prevent both to perform advanced experimental evaluations with humans (which is problematic to assess the robustness and safety of the robot under unpredictable human behaviour), and to envisage short term transfer of these technologies in affordable robots. Another approach, followed by Acroban, is to use components which are at the same time complex, powerful enough, affordable, lightweight and robust, such that it becomes possible to easily design motor primitives/controllers experimentally. This is done at the cost of precision, i.e. these motors have significant backlash, which also makes mathematical modelling nearly intractable. Yet, this can be mitigated and even leveraged by using adequate morphologies and motor primitives which tuning by real world experiments becomes possible thanks to the robustness and light-weightedness of these components. Indeed, Acroban can fall on the ground without breaking itself, and even if it breaks, components can be easily and quickly replaced. As a proof of concept, all movements and motor primitives presented in this article and associated videos were designed by experimental tuning/trialand-error of relatively simple motor primitives that do not rely on any mathematical model of the dynamics of the robot.

In the following section, we present an overview of related work. Then, we outline the mechanical architecture, as well as the mechatronic and control architecture of Acroban. Then, we present stabilization and dynamic walking motor primitives that allow Acroban to keep its balance under (potentially human) external perturbations in a compliant manner. After this, we show that an emergent human-robot interface for leading Acroban by the hand is spontaneously generated and illustrates how morphological computation can be leverated in physical human-robot interaction. Finally, we discuss the positive emotional reactions triggered by Acroban when presented to the general public, children in particular, and in spite of its metallic appearance which constrast strongly with most popular social robots.

This article comes with accompanying videos (also available on http://flowers.inria.fr/acroban.php and discussed in [4]): Video 1: Overview of Acroban:

http://www.youtube.com/watch?v=zHbl-ozA_h0

Video 2: Dynamically controlled mechatronic compliance:

http://www.youtube.com/watch?v=0g72SdIJcaM

Video 3: Intrinsic Structural Compliance:

http://www.youtube.com/watch?v=KjggkAtnn-0

Video 4: Stabilization and robustness to unknown external perturbations:

http://www.youtube.com/watch?v=EENWcI0OIYc

Video 5a: Robust semi-passive dynamic walking:

http://www.youtube.com/watch?v=EKL14Z1weHo

Video 5b: Passive dynamic walking generated by human external perturbation:

http://www.youtube.com/watch?v=gKEjkckxzBU

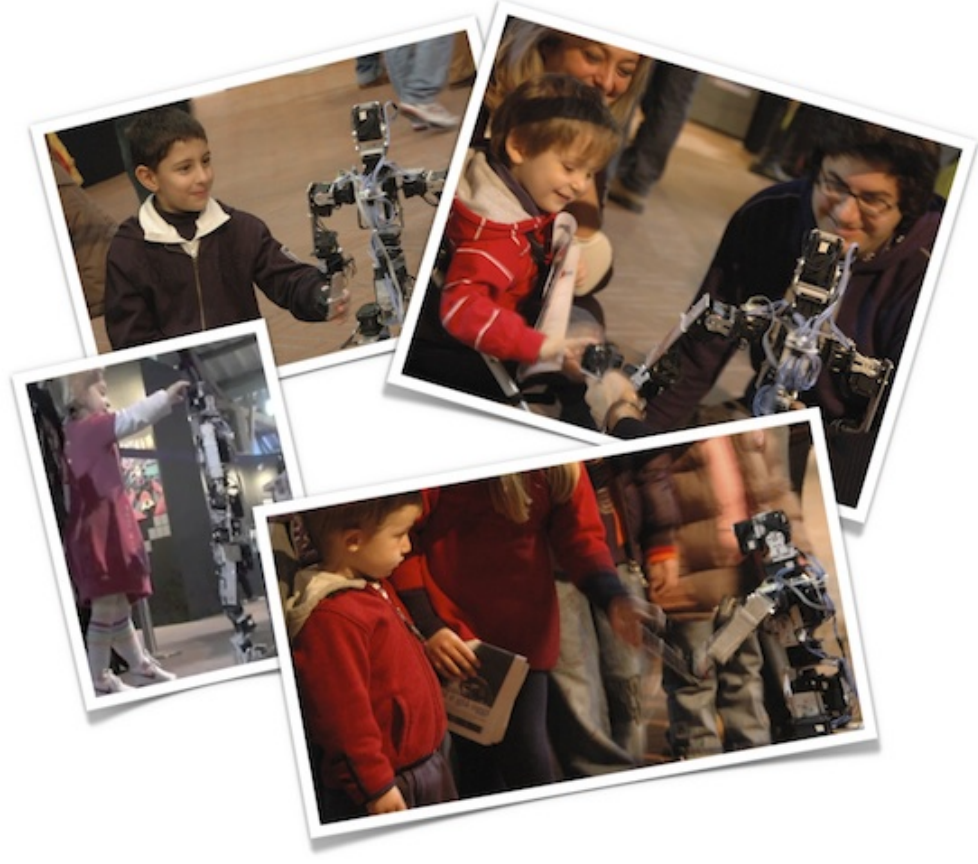

Fig. 1. Robust and playful physical child-robot interaction with Acroban. Around 150 children personnally and continuously interacted with Acroban during a whole week-end in a public space in Citta della Scienza, Napoli, Italy.

Video 6: Guiding Acroban by the hand: an emergent human-robot interface:

http://www.youtube.com/watch?v=5TikvCNkPGA

Video 7: Playful physical child-robot interaction and the Luxo Jr. Effect:

http://www.youtube.com/watch?v=WjAcjxsrV4o

\section{RELATED WORK}

In recent year, several lines of research have flourished around physical human-robot interaction [11]. First of all, researchers have investigated how accidental and/or unwanted contact with robots could be made safe. For instance, the european project Phriends (physical human-robot interaction: dependability and safety) focuses on the robot design and control to allow intrinsically safe physical human-robot interaction (PHRI). They developed a passivity based framework for the control of flexible joint robot. They enabled the DLR lightweight robot arm manipulator to perform compliant manipulation in contact with humans [8] [9]. They also provided keys for systematic evaluation of safety in PHRI. For instance, they investigated real-world threats via collision tests [12]. Similarly, Haddadin et al. studied the problems that can occur due to the physical interaction in human-robot soccer [13].

Physical contact has also been studied in relation to its potentially important communicative function [14]. For instance, sport coaches or dance teachers often use direct touching to modify and correct the student's posture or movement. So, one could imagine using such "communicative" touches to guide or teach robots, such as explored in $[15]-[17]$. 
Physical contact has also been used to program a robot by demonstration. By switching from an active to a passive mode, users can first demonstrate a motion by directly moving the robot and then ask it to reproduce the movement. Hersh et al. manage to make a humanoid reproduce tasks such as putting an object into a box or reaching and grasping an object [18]. Evrard et al. taught a physical collaborative task to a robot: lifting an object by using an indirect demonstration with a human operator [19]. Here, the physical interaction is not direct but through the object. Edsinger et Kemp developed another indirect physical interaction to transfer objects between humans and robots [20]. They showed that without instructions users successfully hand objects to a robot. Furthermore, users naturally tend to orient the object in order to make it match the configuration of the robot's hand and thus simply the grasping.

Physical interaction has been used in particular for tasks like guiding a robot in the environment. For instance, Chen et Kemp proposed a physical interface for a nursing assistant robots. Indeed, people often use direct physical contact to guide a person. Here, they tried to reproduce this interaction to guide a robot. They showed that their interface was more intuitive and efficient than a comparable gamepad interface to guide the robot through a cluttered environment. However, users can not have an analogical control of the robot's direction [21], and the robot is not a full-body biped humanoid. Kosuge et al. developed a dance partner robot composed of an omni directional wheeled base and a body force sensor. The robot is able to coordinate its movements with the human thanks to the physical interaction, i.e. to estimate the next dance step it should perform. However, the step have to be prerecorded [22] [23]. Setiawan et al. realized a human-follow walking by physical interaction. In their work, they split the walking pattern into several units. Then, depending on the forces applied to the hand contact, they can select the next unit needed to walk [10]. In the above mentioned examples, the physical interaction is used to infer the next discrete step or the next discrete command to execute. This symbolic/discretization filters can limit the robustness and fluidity of those systems.

More analogical interactions have also been developed. Driving a Segway can be seen as a fully analogical and physical interaction where users directly grab and orient it to make it move [24]. Similarly, Banks developed the EGGWAY robot which is a mobile platform with a physical interface. Indeed, you can control the robot through direct physical interaction such as pushing or leaning [25]. Boston dynamics developed the robot BigDog, a rough-terrain quadruped robot. This robot can walk and keep its balance even with a priori unknown external forces [26]. Hyon et al. developed a framework for full-body balancing in the presence of external forces such as a physical interaction [27]. Those approaches and robots relied on high-technology highly-precise force controlled actuators (and associated analytical mathematical models), resulting in big heavy fragile robots which cannot easily be experimented "in the wild" interacting with everyday humans, and even less with human children.

Physical interaction is also known to have a strong impact on the user's perception of the human-human interaction and so it will probably be interesting to study its effect on the human-robot interaction. However, few research have been made on this topic. Cramer et al. showed that touch and proactive behavior seems to have a linked effect on the perception of the interaction [28]. Robots such as the Huggable [29], ANTY [30], the Haptic Creature [31], the Paro robot [32] have been developed to further investigate the touch aspect of emotional communication and in particular their effect on hospitalized persons.

\section{THE Design OF ACROBAN}

We here present an overview of Acroban's mechanical structure and controllers. More details are available in [4].

\section{A. Mechanical Structure}

The Acroban platform is a small (about $70 \mathrm{~cm}$ ) and lightweight (about $5 \mathrm{~kg}$ ) humanoid robot with many degrees of freedom (30 dofs) and a multi-articulated spine (see Figure 2 and Video 1). The structure only includes revolute joints, which are all actuated by standard servomotors: Each ankle has 3 joints enforcing a spherical link. Each knee has 1 joint enforcing a revolute link. Each hip has 3 joints enforcing a spherical link. The vertebral column has 5 joints. Each shoulder has 1 joint enforcing a 2-revolute joints link. Each elbow has 2 joints enforcing a 2-revolute joints link. We essentially focused on designing a mechanically rich and open structure in the area of the vertebral column and the pelvis, providing it with 11 degrees of freedom on those areas.

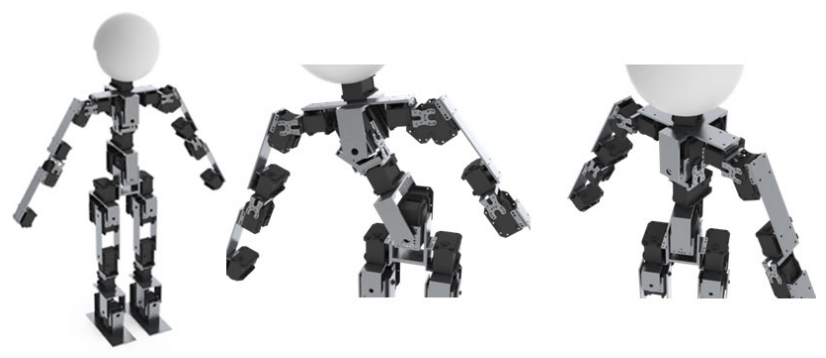

Fig. 2. Acroban Global Structure

Bio-inspired vertebral column. The vertebral column can be viewed as a system linking the pelvis and the shoulder. It enforces two revolutes joints links at its two extremities, each of them providing rotations in the sagittal and the coronal planes and one in the transverse plane, corresponding to the essential degrees of freedom identified in humans during dynamic walking [7]. During movements, and in particular movements related to locomotion, this allows us to get a better independency of the higher part and the lower part of the body. This can also reduce the inertia of the higher part of the body during the gait.

Pelvis. The pelvis, seen as an independent sub-body, produces precise movements of the center of gravity of the robot. It can move by a rotation in the sagittal plane (useful for balance) and in the transverse plane (for the gait). 


\section{B. Mechatronic}

Servomotors. Acroban is actuated by standard servomotors that enforce revolution joints: Robotis' Dynamixe $\left.\right|^{1} \mathrm{RX}-64$ and RX-28. RX-64 (resp. RX-28) can deploy $64 \mathrm{~kg} . \mathrm{cm}^{-1}$ (resp. $28 \mathrm{~kg} . \mathrm{cm}^{-1}$ ) in standing torque, decreased around $30 \mathrm{~kg} . \mathrm{cm}^{-1}$ $\left(15 \mathrm{~kg} . \mathrm{cm}^{-1}\right)$ during movements. They have small backlash and low accuracy due to metal gear reductors, however their mechanic is reversible which allows to get them compliant. The servomotors enforce position control through internal PID controllers. But they also allow adjusting in real time a maximal bound for the torque produced by the joints, and controlling this maximal bound on the torque allows to achieve variable impedance control. A driving mode called "null torque mode" in which the servomotor cancels the rubbing forces of the gears and of the motor to get a completely passive joint is also available and dynamically used.

Springs. Some particular joints (knees, coronal hip and pelvis) are lightly under-powered. We address this problem by using torsion springs as energy accumulator to support servomotors and to introduce compliance also.

Elastics. The use of standard servomotors implies a significant backlash. To address this problem, we use elastics (or extension springs) set between the extremities of the leg which replaces the backlash by a kind of force proportional reaction in the backlash range. Thus, backlash can be leveraged to provide smooth compliance.

\section{Motor Control System}

Servomotors are controlled by a centralized upper layer implementing the logic of movements. This layer is enforced by an embedded system based on ARM technology. The servomotors order refresh frequency is low : $50 \mathrm{~Hz}$. However, each servomotor embeds a control loop of higher frequency (higher than $1 \mathrm{KHz}$ ).

Movements are subdivided into modules called parameterized motor primitives. Motor primitives are combined in order to form global movements of Acroban in a modular way. Motor primitives are closed-loop and read sensor values as inputs (accelerometer, position and position errors of the joint). Movements are constructed by combinations of splines, CPG (Central Pattern Generators) and PID local controler. Outputs are joint position targets, joints maximal torque, and also position in the operational space (cartesian positions of the feets); more complex move are obtained also by taking as output move primitive parameters (typically the gains of splines, or CPG, but also of PID controler).

Finally, we have to consider the direct physical reaction of the mechanical structure of the platform as morphological computation. Indeed, flexible parts (springs and elastics), together with the backlash, produce immediate reactions (typically force proportional reactions) to external perturbations (see http://www.youtube.com/watch?v=KjggkAtnn-0Video 3). Furthermore, the compliance of servo-motors (see Video 2), especially when maximal torque is tuned to be low, allows

\footnotetext{
${ }^{1}$ Robotis. Dynamixel RX64 User's Manual: www.robotis.com
}

the physical interaction between gravity, inertia and Acroban's body to generate movements.

\section{Compliant Stabilizing AND DYNAMIC WALKING}

We present here stabilizing motor primitives aimed at maintaining the system stable under various external mostly unknown perturbations, e.g. a human pushing the robot. In particular, whole body stabilizing motor primitives are designed quasi-independently from the walking pattern. The gait is then designed as a stabilization system perturbed by an active CPG (Central Pattern Generator) coming from the lower part of the body (pelvis and legs).

\section{A. Compliant stabilizing motor primitives}

Compliance. Compliance is twofold: controlled (see Video 2) and structural (see Video 3)). Controlled compliance means adjusting the maximal torque of joints during movements. In a static way, one puts the structure into a configuration with particular morphological computation properties, which can be akin to morphosis [33]. One can simulate for instance an adjustable spring (see e.g. [34]). We use this technique at different levels of the robot: For ankles, in particular to improve feet adherence, for the pelvis and the vertebral column, for arms and shoulders which can set to be even free, and for the sagittal rotation of the pelvis in order to absorb sagittal moment acting on the whole structure.

Structural compliance (i.e. flexibility of the structure, and in particular legs) is also important (see Video 3). It absorbs the impact of foot landing and helps the leg to get locally stable configuration by itself. Quantitatively, along the whole body of Acroban, and when all motors are tuned to be stiff, the flexibility range is approximately 20 degrees as can be seen on Video 3. Let us also mention the slippery sole of Acroban. During the foot landing, it reduces the horizontal component of the impact in a very important way, avoiding important perturbations, and makes the leg find by itself local horizontal adjustment of the foot position. Experiments show that this feature improves significantly the stability of the robot during walk. However, this costs a lack of precision and of efficiency of steps, which adapt this way constantly to the environment.

The higher part of the body (torso, head and arms) makes the center of mass move up (comparing to a body which would have just legs). This makes higher the amplitude of movements of the center of mass in the horizontal plane, and thus makes easier the lateral weight transfer from one leg to the other one. However, at the same time it makes the system more instable. This is mitigated by the vertebral column and the shoulder, which are made compliant and the arms, which are positioned down, making the torso-arm system behave like three coupled pendulums, i.e. the spine as an inverted pendulum to the top of which are attached two pendulums (the arms). For the coronal rotation of the pelvis, i.e. the base of the inverted pendulum, the compliance degree is controlled by its position error (see 
hrefhttp://www.youtube.com/watch?v=EENWcI0OIYcVideo 4).

Experiments show that such a system absorbs shocks and improves the stability of the system. And for some motor primitives that we call semi-passive (in particular the gait, see Section IV-B, the robot uses this pendulum system as local energy accumulator (relying on potential energy). The movement of the pendulum is kept by active movements of legs and pelvis.

Controllers. For the stabilization, compliance is used in conjunction with active controllers: Deformable parallelogram movements on the vertebral column to correct position while bringing minimal moment of the higher part of the body. Pelvis sagittal rotation in order to produce precise movement of the center of mass and also to maintain the pelvis horizontal. Ankle torque in the sagittal plane in order to counter the lean of the robot. By using inverse kinematic of legs, one controls of the higher part of the body in the Cartesian space, and in this way one gets an approximation of Cartesian movements of the center of mass. We use it to absorb horizontal perturbations, but also, via vertical translations, to transform the position of the robot into a minimum of potential energy, and in this way attractor behaviors.

The design of stabilization motor primitives consists in adjusting the compliance of joints and gains of the controllers described above by trial-and-error experiments coupled with expert knowledge.

\section{B. Passive and semi-passive dynamic walking in Acroban}

Passive dynamic walking in Acroban. Considering the stabilizing motor primitives described above, a particular family of external perturbations, i.e. periodic lateral perturbations here generated by a human, provokes spontaneously passive dynamic walking as shown in Video 5b. While the robot uses the same stabilizing motor primitive, this perturbation, amplified by the triple pendulum in the torso, makes it realize dynamic steps forward as a consequence of the mechanical elasticity of the feet and legs. Indeed, during grounded phase, the leg accumulates energy for horizontal translation and torsion moves of the foot; and it frees this energy when the foot is up, making this last one move and perform a step (see Video 5b).

Dynamic Walk as a Stabilized Self-Perturbation. Starting from this intrinsic capability for passive dynamic walking, we designed a motor primitive, based on the use of a CPG directly added on top of the stabilizing motor primitive described above. In this way, one generates dynamic gait by a selfperturbation. In this case, we have what one may call a powered passive walker or a semi-passive walker [35].

The walking loop pattern has two sides: An active side driven by the legs and the pelvis, mixing lateral weight transfer and step forward. This active side, involving only the legs and the pelvis, is generated by CPGs relying on sinusoids. A passive side consisting in the stabilizing motor primitive as described above. The active use of the pelvis, independently of the torso for the step is largely inspired by the human walk (see e.g. [36], [37]).

Besides, it is possible to control the whole walking pattern, i.e. to get the robot turn in all directions and walk forward at various speeds, by controlling the amplitude of its splines, independently for left and right step. This reduces the control of the walk to 2 parameters determining first the sum of the amplitudes of both steps and seconds the relative difference between them.

\section{ROBUSTNESS TO UNKNOWN EXTERNAL PERTURBATION}

As argued in the introduction, most humanoid robots are not robust to unknown potentially large perturbations (e.g. a human giving a push or throwing an object) while they are achieving biped walking, or even only standing up on their two legs. We have conducted a number of experiments to show that Acroban addresses those challenges in a promising manner. We have achieved some quantitative experiments based on the use of repeatable and controllable shocks with objects over Acroban, as well as on the use of a motion tracking system, which gave very positive results. Yet, this kind of experiment, which we will present in a future article, do not really allow to give an assessment of what may happen in less controlled and predictable perturbations, typically those provoked by physical contact with a human. This is why we present here, in the form of videos, more qualitative results exploring a large variety of external perturbations.

Postural stabilization. First, Video 4, which deliberately includes long sequence shots with no editing to show the robustness of the displayed capabilities, shows Acroban stabilization abilities in the face of external perturbations and while standing up without walking. Acroban is able to maintain its balance when a real football ball (very big and rather heavy as compared to its own size and weight) is thrown at him at non negligible speeds and at various places of its body as well as many impact angles. Furthermore, Acroban is able to maintain its postural balance when a human pushes or pulls many parts of its body and without anticipation (there are no sensors allowing the robots to sense and prepare for external impacts). It is also possible to take Acroban by the shoulders, balance its whole body, and then literally throw it on the floor: if the throwing is reasonable, landing happens rather smoothly, the shock is absorbed thanks to the structure compliance, and Acroban stays stood up (see video). Finally, as shown at the end of Video 4, we even tried to launch Acroban on a skateboard, and this resulted again in quite robust ability to keep its balance.

Robustness to external perturbations while dynamically walking. As explained above, dynamic walking in Acroban is the result of combining postural stabilization with a selfperturbation in the form of a CPG on the legs, dynamically transfering weight from one leg to the other. As shown in Video 5a, Video 5a, Acroban still keeps its balance when in addition a football ball is thrown at him while dynamically walking. The same holds when pushed by a human at various positions of its body. When put on a skate-board pushed by 
a human, Acroban robustly oscillates its legs without falling. When put on a gentle downward or upward slope, Acroban continues to be able to go forward without falling.

\section{EMERgEnt PhysicAl Human-Robot InTERfACE: LEAD ME BY THE HAND}

Previous section showed that Acroban was capable of maintaining its balance and absorbing shocks while walking under potentially unwanted or accidental or "wanted but for kidding the robot" unknown human generated perturbations. In this section, we show that those capabilities can be leveraged to generate original kinds of human-robot interfaces.

Leading Acroban by the hand. An original and initially unplanned feature of Acroban appeared during a public demonstration of the robot in Napoli's Science Museum in Italy in november 2009 (see Video 7), where numerous children (around 150) could interact personnally and physically with the robot ${ }^{2}$ While showing that Acroban could be pushed or pulled at various places of its body (head, torso, legs, arms, ...) without falling and keeping natural smooth compliant movements, some children began to take the hands of Acroban, like parents take the hands of their children, and tried to have Acroban follow them. And, to our surprise, Acroban followed. As we afterwards robustly and easily reproduced as shown at the end of Video 6, they could lead Acroban by the hand in any direction, have it turn on itself, and this even by applying extremely gentle forces on the hands or if only one hand was taken. Yet, no force sensors to measure such external perturbations are used and not a single line of code in Acroban was written to produce such a behaviour.

A posteriori, this phenomenon can be understood in the following manner. This capability of Acroban appears when Acroban oscillates its legs with the CPG as described in section 5, tuned such that neither translation nor rotation of its whole body happen, and coupled with the postural stabilization motor primitive. As explained earlier, this CPG acts as a selfperturbation which is continuously counterbalanced by the stabilization motor primitive. Parameters of this CPG allow us to control slight changes in the geometry (mainly in the legs), distribution of weight and dynamics of the robot in this situation, which in turn make the robot fall into various movement attractors such as going forward, turning, or standing still while transferring weight from one leg to the other. Similarly, when a human takes the hands of Acroban, and due to the compliance of its torso in particular, this produces 1) obviously a modification of the geometry of the arms, but also 2) a modification of the geometry of the multi-articulated torso. As a consequence, this also produces a modification of the weight distribution. And this leads to a changed global movement attractor, which may be conceptualized as minimizing the energy of the whole (robot, human perturbation) system, making the robot follow the human in a way which approximately cancels the human pull forces, and thus allows

\footnotetext{
${ }^{2}$ Interaction with Acroban happened continuously during a whole weekend, i.e. twice 8 hours in a row, which also shows the mechanical robustness of Acroban
}

the human to drive Acroban by applying very low forces on the hand.

Morphological computation. It is important to note that here the code/algorithms that lead to this behaviour are strictly the superposition of the compliant stabilizing motor primitive and the CPG self-perturbation allowing the robot to walk dynamically. There is no such thing as bits of algorithms like "if certain forces $\mathrm{F}$ are sensed in the arm, then change the parameters $\mathrm{P}$ of the motor primitives in order to walk in direction D". The human taking the hand is for the system no different than any other unknown external perturbation, and of course the system has no notion of "other" or "human". But the particular structural properties of this specific kind of perturbation in relation with the specific human-like morphology of the robot generate spontaneously a behaviour which can be interpreted as "following the human in the direction he seems to indicate by pulling my hands" by an external observer. Replacing potentially rather complex lines of codes and algorithmic computation if one would have liked to implement this kind of behaviour in a classical engineering approach, this consequence of the laws of physics and associated dynamical systems is a typical illustration of what has been termed "morphological computation" [5]. In addition, it might be fair to call this a truly emergent humanrobot interface.

Insights on human locomotion. Another interesting feature of this phenomenon is that it is mainly a change in the geometry and distribution of weight in the multi-articulated human-inspired torso that allows to drive the walking of the robot. This resonates with recent novel approaches to the understanding of human locomotion that proposed, in contrast to a very large litterature focusing on the role of the legs and their coordination with the head posture/gaze [38], that the trunk itself may have a central role in both initiating and directing human dynamic walking [7] (and not unlike taught for learning certain sports such as parallell skiing driven by the trunk).

\section{COMPLIANCE AND FURTHER INTERFACES}

In the previous section, we have shown how an emergent non-trivial interface could be generated for free with the natural compliant and dynamical properties of the robot. Of course, it is also possible to leverage this compliance and approximate force control to implement more traditional kinds of human-robot interfaces. Indeed, compliance can allow joints to become a tangible interface, and the whole body becomes a haptic interface. A human can modify the state (joint position) of the robot by a direct compliant physical manipulation, making possible the exchange of analog information: those state changes can be measured and specifically be interpreted as commands that trigger specific algorithms in the robot. As the compliance/resistance of each actuator can be controlled dynamically, and as forces can be indirectly sensed through position errors of the low-level PID controllers, it is possible to devise fluid interactions where for example the robot is doing its own movements with its arms tuned stiff, and as a human 
takes one of its arms, a resistance is immediately sensed and the arms become possibly completely free to be manipulated by the human (see Video 1 and Video 7). In this interaction mode where a human can take the arm of the robot, it is also possible to devise interactions such as controlling one part of the body (e.g. left arm) with another part of the body (e.g. righ arm), see Video 7

\section{EMOTIONS IN PHYSICAL HUMAN ROBOT INTERACTION WITH ACROBAN}

While demonstrating Acroban with the wide audience public exhibition Futuro Remoto in Citta della Scienza, Napoli, Italy in November 2009 Video 7), we discovered that Acroban provokes spontaneous highly positive emotional reactions. Yet, as opposed to many other robots, its morphology is neither roundish nor cute. He has no big eyes and no funny color. $\mathrm{He}$ even only has three raw motors in place of the head. He is just made of metal, and its appearance shows it explicitly. At first glance, its visual appearance creates low expectation of intelligence and life-likeness. But when it begins to move with smooth and compliant movements and one can touch it, its life-like natural dynamics triggers a high contrast and positive surprise, which may explain the highly positive emotional reactions observed. Life unexpectedly appears out of a neutral metallic object, a bit like what happens in Pixar's Luxo Jr animated cartoons where a neutral desk lamp provokes a positive surprise when animated like a life form [39]. Similarly, the animation of Acroban, leveraging semi-passive dynamics and gravity like legged animals, follows principles of animated cartoons to trigger the illusion of life: in particular, its highcompliance and passive dynamics allow observers to directly see/feel the weight and inertia of the robot, much like Disney's explanations on the "importance of weight" for animating walking creatures [40] (see also [41] for a related study). In addition to animated cartoon characters, Acroban affords the possibility of fluid physical interaction, which enhances this illusion of life. These observations with Acroban were confirmed qualitatively in more recent public demonstrations, including a public multimedia fair in Los Angeles [3].

In spite of those strong positive emotional reactions, in particular in children, Acroban violates most of the recent tendencies about visual design of social personal robots. These tendencies advocate the use of morphologies / shape / colors which should trigger positive expectations before the interaction starts, for example by taking baby-like features, of course within a certain limit in order to avoid the socalled "uncanny valley" effect [42]. But these approaches, while preparing the mind of the user positively, also take the risk to frustrate human users if the robot afterwards does not meet their expectations. On the contrary, Acroban suggests an approach where the design should on the contrary minimize expectations, and thus maximize the effect of positive surprise due to actual behaviour when the user discovers that the robots is actually "more" that it seemed at first sight. Of course, this effect is at this stage still a speculation which should now be investigated in detail with Acroban in comparison with other more mainstream robots and using systematic quantitative metrics in controlled HRI experiments. In particular, in addition to quantify precisely this effect, it will be of high interest to try to disentangle the respective roles of animation per se and of the capacity to touch and physically interact with the robot.

\section{CONCLUSION}

Acroban allows unique whole-body robust, natural and intuitive physical interaction with both adults and children, thanks to the combination of of several properties: 1) it is whole-body compliant thanks to variable impedance control and also thanks to the use of elastics and springs; 2) it has a bio-inspired vertebral column allowing more flexibility in postural and equilibrium control; 3) it is light-weight; 4) it has simple low-level controllers that leverage the three first properties. Moreover, the capabilities for physical human-robot interaction that we have shown are not using a model of the human, and in this sense are "model free": 1) the capability of the robot to keep its equilibrium while being manipulated or pushed by humans is a result of the intrinsic capability of the whole body to absorb unpredicted external perturbations; 2) the capability of leading Acroban by the hand is an emergent human-robot interface made possible by the self-organizing properties of the body and its low-level controllers and was observed a posteriori only after the robot was conceived and without any initial plan to make this possible. In this article, those many properties were shown qualitatively in order to provide a global overview of how this general approach allows to design robust and natural physical human-robot interaction. This of course needs to be complemented by more systematic and more quantitative studies of both Acroban's robustness and of the properties of physical human-robot interaction, which shall be achieved in future work.

Finally, an originality of Acroban, especially as opposed to other humanoid robots capable of dynamic walking and advanced motor control, such as ASIMO or HRP, is that is is made with relatively low-cost components which lack of precision is counterbalanced with the robustness due to global geometry and compliance. This shows that stimulating and novel design opportunities could be explored in the future and be very useful to the development of personal robotics in our society.

Acknowledgement We would like to thank Matthieu Lapeyre and Jérome Béchu, in particular for their important help for setting up experiments. This research is partially funded by ERC Starting Grant EXPLORERS 240007.

\section{REFERENCES}

[1] K. Hirai, M. Hirose, Y. Haikawa, and T. Takenaka, "The development of Honda humanoid robot," in IEEE International Conference on Robotics and Automation. INSTITUTE OF ELECTRICAL ENGINEERS INC (IEEE), 1998, pp. 1321-1326.

[2] K. Kaneko, F. Kanehiro, M. Morisawa, K. Miura, S. Nakaoka, and S. Kajita, "Cybernetic human HRP-4C", in 9th IEEE-RAS International Conference on Humanoid Robots, 2009. Humanoids 2009, 2009, pp. $7-14$.

[3] O. Ly and P.-Y. Oudeyer, "Acroban the humanoid: playful and compliant physical child-robot interaction," in ACM Siggraph Emerging Technologies, 2010, pp. 1-1. 
[4] O. Ly, M. Lapeyre, and P.-Y. Oudeyer, "Bio-inspired Vertebral Column, Compliance and Semi-Passive Dynamics in a Lightweight Robot," in IEEE/RSJ International Conference on Intelligent Robots and Systems (IROS 2011), 2011.

[5] C. Paul, M. Lungarella, and F. Iida, "Morphology, control and passive dynamics," Robotics and Autonomous Systems, vol. 54, no. 8, pp. 617618, 2006.

[6] R. Pfeifer, M. Lungarella, and F. Iida, "Self-organization, embodiment, and biologically inspired robotics," Science, vol. 318, no. 5853, p. 1088, 2007.

[7] J. Ceccato, M. de Seze, C. Azevedo, and J. Cazalet, "Comparison of trunk activity during gait initiation and walking in humans," PLoS One, vol. 4, no. 12, 2009.

[8] Albu-Schaffer, C. Ott, and G. Hirzinger, "A Unified Passivity-based Control Framework for Position, Torque and Impedance Control of Flexible Joint Robots," The International Journal of Robotics Research, vol. 26, no. 1, pp. 23-39, Jan. 2007. [Online]. Available: http://ijr.sagepub.com/cgi/doi/10.1177/0278364907073776

[9] A. Albu-Schaffer, O. Eiberger, M. Grebenstein, S. Haddadin, C. Ott, T. Wimbock, S. Wolf, and G. Hirzinger, "Soft robotics," IEEE Robotics \& Automation Magazine, vol. 15, no. 3, pp. 20-30, Sep. 2008. [Online]. Available: http://ieeexplore.ieee.org/lpdocs/epic03/ wrapper.htm?arnumber $=4624580$

[10] S. Setiawan, J. Yamaguchi, and a. Takanishi, "Physical interaction between human and a bipedal humanoid robot-realization of humanfollow walking," Proceedings 1999 IEEE International Conference on Robotics and Automation (Cat. No.99CH36288C), pp. 361367, 1999. [Online]. Available: http://ieeexplore.ieee.org/lpdocs/epic03/ wrapper.htm?arnumber $=770005$

[11] a. Desantis, B. Siciliano, a. Deluca, and a. Bicchi, "An atlas of physical humanrobot interaction," Mechanism and Machine Theory, vol. 43, no. 3, pp. 253-270, Mar. 2008. [Online]. Available: http://linkinghub.elsevier.com/retrieve/pii/S0094114X07000547

[12] S. Haddadin, a. Albu-Schaffer, and G. Hirzinger, "Requirements for Safe Robots: Measurements, Analysis and New Insights," The International Journal of Robotics Research, vol. 28, no. 11-12, pp. 1507-1527, Aug. 2009. [Online]. Available: http://ijr.sagepub.com/cgi/ doi/10.1177/0278364909343970

[13] S. Haddadin, T. Laue, U. Frese, S. Wolf, A. Albu-Schäffer, and G. Hirzinger, "Kick it with elasticity: Safety and performance in humanrobot soccer," Robotics and Autonomous Systems, vol. 57, no. 8, pp. 761-775, Jul. 2009. [Online]. Available: http://linkinghub.elsevier. com/retrieve/pii/S0921889009000530

[14] M. J. Hertenstein, "Touch: Its Communicative Functions in Infancy," Human Development, vol. 45, no. 2, pp. 70-94, 2002. [Online]. Available: http://www.karger.com/doi/10.1159/000048154

[15] T. Tsuji and T. Ito, "Command recognition by haptic interface on human support robot," 2009 IEEE/RSJ International Conference on Intelligent Robots and Systems, pp. 3178-3183, Oct. 2009. [Online]. Available: http://ieeexplore.ieee.org/lpdocs/epic03/wrapper. htm?arnumber $=5354562$

[16] F. Dalla Libera, T. Minato, I. Fasel, H. Ishiguro, E. Pagello, and E. Menegatti, "A new paradigm of humanoid robot motion programming based on touch interpretation," Robotics and Autonomous Systems, vol. 57, no. 8, pp. 846-859, Jul. 2009. [Online]. Available: http://linkinghub.elsevier.com/retrieve/pii/S092188900900061X

[17] T. Minato, Y. Yoshikawa, T. Noda, H. Ishiguro, and M. Asada, "CB2: A child robot with biomimetic body for cognitive developmental robotics," 2007 7th IEEE-RAS International Conference on Humanoid Robots, pp. 557-562, Nov. 2007. [Online]. Available: http://ieeexplore. ieee.org/lpdocs/epic03/wrapper.htm?arnumber $=4813926$

[18] M. Hersch, F. Guenter, S. Calinon, and A. Billard, "Dynamical System Modulation for Robot Learning via Kinesthetic Demonstrations Dynamical System Modulation for Robot Learning via Kinesthetic Demonstrations," IEEE Transactions on Robotics, 2008.

[19] P. Evrard, E. Gribovskaya, S. Calinon, A. Billard, and A. Kheddar, "Teaching physical collaborative tasks: object-lifting case study with a humanoid," 2009 9th IEEE-RAS International Conference on Humanoid Robots, pp. 399-404, Dec. 2009. [Online]. Available: http: //ieeexplore.ieee.org/lpdocs/epic03/wrapper.htm?arnumber=5379513

[20] A. Edsinger and C. C. Kemp, "Human-Robot Interaction for Cooperative Manipulation: Handing Objects to One Another," RO-MAN 2007 - The 16th IEEE International Symposium on Robot and Human Interactive
Communication, pp. 1167-1172, 2007. [Online]. Available: http: |//ieeexplore.ieee.org/lpdocs/epic03/wrapper.htm?arnumber=4415256

[21] T. L. Chen and C. C. Kemp, "Lead me by the hand: Evaluation of a direct physical interface for nursing assistant robots," in 2010 5th ACM/IEEE International Conference on Human-Robot Interaction (HRI). IEEE, Mar. 2010, pp. 367-374.

[22] K. Kosuge, T. Hayashi, Y. Hirata, and R. Tobiyama, "Dance Partner Robot -Ms DanceR-," Proceedings of the Conference on Intelligent Robots and Systems, 2003.

[23] T. Takeda, Y. Hirata, and K. Kosuge, "HMM-based Error Recovery of Dance Step Selection for Dance Partner Robot," Proceedings 2007 IEEE International Conference on Robotics and Automation, pp. 1768-1773, Apr. 2007. [Online]. Available: http://ieeexplore.ieee.org/ lpdocs/epic03/wrapper.htm?arnumber $=4209342$

[24] H. G. Nguyen, "Segway robotic mobility platform," Proceedings of SPIE, no. February 2002, pp. 207-220, 2004. [Online]. Available: http://link.aip.org/link/?PSI/5609/207/1\&Agg=doi

[25] J. L. Banks, "EGGWAY: A mobile platform with an intuitive physical interface," Artificial Intelligence, 2005.

[26] M. Raibert, K. Blankespoor, G. Nelson, and R. Playter, "BigDog, the Rough-Terrain Quadruped Robot," Control, pp. 6-9, 2008.

[27] S.-H. Hyon, J. Hale, and G. Cheng, "Full-Body Compliant HumanHumanoid Interaction: Balancing in the Presence of Unknown External Forces," IEEE Transactions on Robotics, vol. 23, no. 5, pp. 884-898, Oct. 2007. [Online]. Available: http: //ieeexplore.ieee.org/lpdocs/epic03/wrapper.htm?arnumber=4339533

[28] H. S. Cramer, N. a. Kemper, A. Amin, and V. Evers, "The effects of robot touch and proactive behaviour on perceptions of humanrobot interactions." Proceedings of the 4th ACM/IEEE international conference on Human robot interaction - HRI '09, p. 275, 2009. [Online]. Available: http://portal.acm.org/citation.cfm?doid=1514095. 1514173

[29] W. Stiehl, J. Lieberman, C. Breazeal, L. Basel, R. Cooper, H. Knight, L. Lalla, a. Maymin, and S. Purchase, "The huggable: a therapeutic robotic companion for relational, affective touch," CCNC 2006. 2006 3rd IEEE Consumer Communications and Networking Conference, 2006., pp. 1290-1291, 2005. [Online]. Available: http: //ieeexplore.ieee.org/lpdocs/epic03/wrapper.htm?arnumber=1593253

[30] J. Saldien, K. Goris, B. Vanderborght, B. Verrelst, R. V. Ham, and D. Lefeber, "ANTY : The development of an intelligent huggable robot for hospitalized children," Development, 2006.

[31] S. Yohanan and K. E. Maclean, "The Haptic Creature Project : Social Human-Robot Interaction through Affective Touch," Development, pp. $1-5,2010$.

[32] K. Wada and T. Shibata, "Robot Therapy in a Care House - Its Sociopsychological and Physiological Effects on the Residents -," Science And Technology, no. May, pp. 3966-3971, 2006.

[33] D. Christensen, U. Schultz, and K. Stoy, "A distributed strategy for gait adaptation in modular robots," in Robotics and Automation (ICRA), 2010 IEEE International Conference on. IEEE, 2010, pp. 2765-2770.

[34] S. Peter, S. Grimmer, S. Lipfert, and A. Seyfarth, "Variable joint elasticities in running," Autonome Mobile Systeme 2009, pp. 129-136, 2009.

[35] S. Collins, A. Ruina, R. Tedrake, and M. Wisse, "Efficient bipedal robots based on passive-dynamic walkers," Science, vol. 307, no. 5712, p. 1082, 2005.

[36] C. Vaughan, "Theories of bipedal walking: an odyssey," Journal of biomechanics, vol. 36, no. 4, pp. 513-523, 2003.

[37] P. Rodman and H. McHenry, "Bioenergetics and the origin of hominid bipedalism," American Journal of Physical Anthropology, vol. 52, no. 1, pp. 103-106, 1980.

[38] J. Rose and J. Gamble, Human walking. Lippincott Williams \& Wilkins, 2006.

[39] S. Mealing, The Art and Science of Computer Animation. Intellect Books, 1998.

[40] F. Thomas and O. Johnston, The Illusion of life: Disney animation. Hyperion, 2001.

[41] T. Chaminade, J. Hodgins, and M. Kawato, "Anthropomorphism influences perception of computer-animated characters'actions," Social Cognitive and Affective Neuroscience, vol. 2(3), pp. 206-216, 2007.

[42] M. Mori, "Bukimi no tani," Energy, vol. 7, no. 4, pp. 33-35, 1970. 